\title{
VUV spectroscopy of carbon dust analogs: contribution to interstellar extinction
}

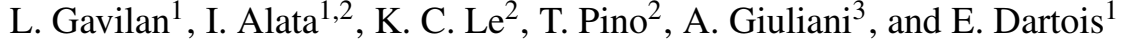 \\ ${ }^{1}$ Institut d'Astrophysique Spatiale (IAS), CNRS, Univ. Paris Sud, Université Paris-Saclay, 91405 Orsay, France \\ e-mail: lisseth.gavilan@ias.u-psud.fr \\ 2 Institut des Sciences Moléculaires d'Orsay (ISMO), CNRS, Univ. Paris Sud, Université Paris-Saclay, 91405 Orsay, France \\ 3 DISCO beam line, SOLEIL synchrotron, 91190 Saint-Aubin, France
}

Received 31 July 2015 / Accepted 13 November 2015

\begin{abstract}
Context. A full spectral characterization of carbonaceous dust analogs is necessary to understand their potential as carriers of observed astronomical spectral signatures such as the ubiquitous UV bump at $217.5 \mathrm{~nm}$ and the far-ultraviolet (FUV) rise common to interstellar extinction curves.

Aims. Our goal is to study the spectral properties of carbonaceous dust analogs from the FUV to the mid-infrared (MIR) domain. We seek in particular to understand the spectra of these materials in the FUV range, for which laboratory studies are scarce.

Methods. We produced analogs to carbonaceous interstellar dust encountered in various phases of the interstellar medium: amorphous hydrogenated carbons (a-C:H), for carbonaceous dust observed in the diffuse interstellar medium, and soot particles, for the polyaromatic component. Analogs to a-C:H dust were produced using a radio-frequency plasma reactor at low pressures, and soot nanoparticles films were produced in an ethylene $\left(\mathrm{C}_{2} \mathrm{H}_{4}\right)$ flame. We measured transmission spectra of these thin films (thickness $<100 \mathrm{~nm}$ ) in the far-ultraviolet (190-250 nm) and in the vacuum-ultraviolet (VUV; 50-190 nm) regions using the APEX chamber at the DISCO beam line of the SOLEIL synchrotron radiation facility. These were also characterized through infrared microscopy at the SMIS beam line.

Results. We successfully measured the transmission spectra of these analogs from $\lambda=1 \mu \mathrm{m}$ to $50 \mathrm{~nm}$. From these, we extracted the laboratory optical constants via Kramers-Kronig inversion. We used these constants for comparison to existing interstellar extinction curves.

Conclusions. We extend the spectral measurements of these types of carbonaceous analogs into the VUV and link the spectral features in this range to the $3.4 \mu \mathrm{m}$ band. We suggest that these two materials might contribute to different classes of interstellar extinction curves.
\end{abstract}

Key words. dust, extinction - infrared: ISM - ultraviolet: ISM - methods: laboratory: solid state - galaxies: ISM

\section{Introduction}

\subsection{General overview}

Carbon-based materials are abundant in circumstellar environments and in the diffuse interstellar medium (DISM; van Diedenhoven et al. 2004; Henning \& Salama 1998; Greenberg \& Li 1999; Draine 2003; Jäger et al. 2011). They are mainly produced in the envelopes of asymptotic giant branch (AGB) stars (Gail et al. 2009; Molster \& Waters 2003) and are injected into the DISM. Carbonaceous dust can then be processed (by photons, ions, cosmic rays) and can further evolve during their lifetime in these heterogeneous environments (Jones et al. 2014). Carbon materials can be classified according to their sizes, which range from (large) polycyclic hydrocarbon molecules to solid-state carbon grains, their aliphatic vs. aromatic content, and their spectra, among other properties (e.g., Henning \& Salama 1998). In solid carbon, $\mathrm{sp}^{2}, \mathrm{sp}^{3}$, and mixed hybridizations are possible, giving rise to materials with different short-, medium-, and long-range orders (Jäger et al. 1998; Papoular et al. 1996; Tielens 2008).

Different carbon dust components in space are identified by their observed spectral signatures. In the infrared, observations of the $3.4 \mu \mathrm{m}$ absorption band were attributed to vibrational $\mathrm{CH}_{2}$ and $\mathrm{CH}_{3}$ aliphatic modes in carbonaceous materials. The counterparts of the $3.4 \mu \mathrm{m}$ band at $6.85 \mu \mathrm{m}$ and $7.25 \mu \mathrm{m}$ were later observed by space telescopes. The carriers of these bands were named HAC by the astrophysical community (e.g., Pendleton \& Allamandola 2002; Furton et al. 1999; Scott et al. 1997; Duley 1994). The HAC denomination for laboratory analogs has been used for a wide variety of hydrocarbon solids, even though the structure of these materials varies from amorphous to disordered carbons.

In the following, we refer to the plasma-produced analogs as $\mathrm{a}-\mathrm{C}: \mathrm{H}$, a term used in the physics community. Amorphous refers to the lack of order at any scale in these materials. The laboratory infrared spectra of these materials match observed spectra, particularly regarding the shape and relative strength of the $3.4 \mu \mathrm{m}$ band (Dartois et al. 2007). The structure associated with the hydrogen-rich a-C:H materials is characterized by few aromatic units linked in a mainly aliphatic backbone (e.g., Fig. 5 of Dartois et al. 2005).

Some astrophysical carbon materials subjected to UV light re-emit in the mid-infrared. Observations of these emission features, called AIB for aromatic infrared bands, appear 
consistently between 3 to $20 \mu \mathrm{m}$ and have been classified into three main classes (Peeters et al. 2002). The intimate structures of the carriers of these different classes of AIBs are still unknown, although they are probably built on a polyaromatic skeleton spanning sizes from large molecules to small nanograins (Leger \& Puget 1984; Allamandola et al. 1985; Goto et al. 2007; Sloan et al. 2007; Pino et al. 2008; Tielens 2008; Joblin \& Tielens 2011; Li \& Draine 2012; Carpentier et al. 2012; Yang et al. 2013).

Interstellar extinction curves, derived from observations of several lines of sight from the near-IR to the UV, provide another clue to the type of dust present in the ISM. A persistent spectral feature is the strong absorption bump at $4.6 \mu \mathrm{m}^{-1}$ $(217.5 \mathrm{~nm})$, discovered by Stecher \& Donn (1965), whose central position is surprisingly constant over several lines of sight, and whose origin is still debated (Fitzpatrick \& Massa 2005, 2007). A steep far-ultraviolet (FUV) rise also characterizes many of these curves, tracing extinction variations. The lack of correlation between the spectral properties of the UV bump and the relative strengths of the bump and the FUV rise points to different types of dust carriers (Greenberg \& Chlewicki 1983; Fitzpatrick \& Massa 2007). Graphite particles were initially proposed as the carrier of the UV bump by Draine \& Lee (1984). More recently, hydrogenated carbons were proposed as potential carriers (Colangeli et al. 1997; Schnaiter et al. 1998; Mennella et al. 1999; Jäger et al. 2008), supporting theoretical work by Duley \& Seahra (1998).

The central goal of this study is to obtain absolute spectral properties of carbon dust analogs in both the $\mathrm{VUV} / \mathrm{Vis}$ regions and the infrared ranges, complementing earlier studies of hydrocarbon solids, particularly in the VUV range, where optical measurements are scarce. A full spectral characterization of these materials will clarify their potential contributions as carriers of observed spectral features in the infrared and in the UV, such as the FUV rise and also the $217.5 \mathrm{~nm}$ bump of interstellar extinction curves. In addition, infrared spectra allow us to characterize the quality and suitability of our samples as interstellar analogs. These transmission spectra allow the derivation of optical constants from these carbonaceous materials, necessary to simulate observed astronomical extinction curves.

Carbon analogs have been produced in the laboratory using different methods and their spectra in transmission, reflection, or electron energy loss spectroscopy (EELS; see Henning et al. 2004 for a review) has been used to extract optical constants. Stecher \& Donn (1965) first derived optical constants of nanometer-sized graphitic particles. Draine \& Lee (1984) derived the optical constants from laboratory and observational data for graphite down to $80 \mathrm{~nm}$ by the Kramers-Kronig approach using EELS data from Tosatti \& Bassani (1970). Zubko et al. (1996) used the same method to obtain the optical constants of several solid hydrocarbons from the millimeter to the FUV down to $40 \mathrm{~nm}$. Schnaiter et al. $(1996,1998)$ derived the optical constants through UV measurements of nano-sized carbon grains using the matrix-isolation technique in the $200-400 \mathrm{~nm}$ region. Furton et al. (1999) also obtained the optical constants of a hydrogenated carbon film by trial and error fitting in the range of 300-1000 nm. These values have a dispersion of between $\sim 10 \%$ and $20 \%$ at any given wavelength, and they compare closely to other published data for similar a-C:H materials, for example, Smith (1984) between 253-900 nm, and for organic residues, for example, Jenniskens (1993) from $115 \mathrm{~nm}$ to $1.7 \mu \mathrm{m}$. Jäger et al. (1998) derived the optical constants of carbonized cellulose samples using the Lorentz-oscillator fit method in the range of 200-500 $\mathrm{nm}$ based on reflectance measurements. This was done later for gas-phase condensed soots from $120 \mathrm{~nm}$ to the mid-infrared (Jäger et al. 2008). Few laboratory based determinations of optical constants of carbon analogs exist for $\lambda<120 \mathrm{~nm}$. Fink et al. (1984) measured the EELS spectra from $300 \mathrm{~nm}$ to $40 \mathrm{~nm}$ for amorphous carbons. Colangeli et al. (1993) measured the spectra of carbon samples down to $\sim 50 \mathrm{~nm}$ through transmission or reflection on a gold-coated quartz window.

This paper is organized as follows. In Sect. 2 we describe the experimental setups used in sample preparation and characterization of the carbonaceous materials. In Sect. 3 we present the UV and MIR spectroscopy of both materials. In Sect. 4 we describe the method used to derive the optical constants. In Sect. 5 we discuss the astrophysical implications of these measurements. Finally, in Sect. 6 we present our conclusions.

\section{Experiments}

We studied two types of carbonaceous dust: a-C:Hs and soot samples. The a-C:Hs were produced using an R.F. plasma reactor at low pressures. Their typical structural unit is shown in Dartois et al. (2005). Soot materials have been produced in an ethylene $\left(\mathrm{C}_{2} \mathrm{H}_{4}\right)$ flame and provide samples dominated by a polyaromatic carbon skeleton (e.g., Carpentier et al. 2012). While our a-C:H materials have a high $\mathrm{H} / \mathrm{C}$, our soot materials have low $\mathrm{H} / \mathrm{C}$.

Two types of substrates were used: $\mathrm{MgF}_{2}$ windows and TEM grids (Ted Pella, Inc.) covered with a nm-thick metallic or carbon membrane. Samples of $\mathrm{MgF}_{2}$ were used to ensure UV-visible transmission measurements down to $\lambda=120 \mathrm{~nm}$. Samples deposited on the $3 \mathrm{~mm}$ TEM grids were used for transmission measurements from $\lambda=120 \mathrm{~nm}$ to $50 \mathrm{~nm}$. The grids were 300 mesh $\mathrm{Au}$ or $\mathrm{Cu}$ substrates covered with a 2-3 nm layer of gold or platinum, or 10-25 nm of carbon. Manipulation of the TEM grids was done with high-precision tweezers, but more often than not, the fragile grid supports were bent and/or the thin membrane shattered, either during installation in the sample holder or during deposition of the film. We inspected the films with a binocular microscope at the Institut d'Astrophysique Spatiale and with microscopic images taken at the SMIS beam line at the SOLEIL synchrotron.

As a result of four experimental campaigns in 2014 and 2015 at the DISCO VUV beam line of the SOLEIL synchrotron, we found that while Au or Pt membranes (2-3 nm) had good transmittance in the VUV $(T \leq 30 \%)$, they were very fragile during manipulation and during film deposition. Most VUV measurements on these substrates showed that the membranes were damaged either partially or completely. Instead, the carbon membranes on TEM grids $(T \leq 20 \%)$ proved to be more robust. We discuss the effect of these substrates on the film transmission spectra in Appendix B.

\subsection{Preparation of a-C:H films}

SICAL-ICP is an experimental setup developed for the purpose of producing hydrogenated amorphous carbon films, see Fig. 1. It consists of an inductively coupled radio frequency (R.F.) generator working at $13.56 \mathrm{MHz}$ (Elite series, MKS) with a dynamic range of $1-600 \mathrm{~W}$. A background pressure of $<0.01 \mathrm{mbar}$ is maintained by a primary pump. The precursor hydrocarbon gas $\left(\mathrm{CH}_{4}\right)$ supply is manually controlled with a fine valve and deposits are made in continuous flow kept at $\sim 0.1$ mbar, after passivation of the chamber with the precursor.

The R.F. discharge is operated in a glass tube (length = $\left.1 \mathrm{~m}, \phi_{\mathrm{ext}}=65 \mathrm{~mm}\right)$, allowing for the plasma to symmetrically 


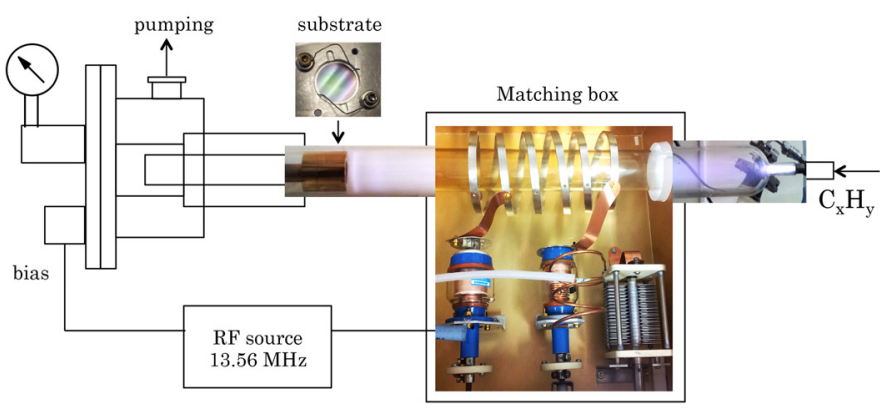

Fig. 1. SICAL- ICP is a setup allowing plasma-induced chemical vapor deposition. It is comprised of a glass tube in which an inductively coupled plasma (ICP) on a continuum hydrocarbon gas flow is maintained by an R.F. source. The plasma envelopes the sample holder placed near the matching box.

envelop the sample holder. The R.F. power is inductively coupled to the plasma by means of an impedance matching network linked to a copper coil around the glass cylinder. The tuning box allows extracting a portion of the R.F. power coupled to the substrate holder acting as an externally applied bias. For most samples, the power was kept at $100 \mathrm{~W}$, with $5 \mathrm{~W}$ reflected. The sample holder consists of a gold-coated copper block capable of holding $20 \mathrm{~mm}$ windows. The holder can be internally cooled by water, and the substrate can be installed by removing a mechanical cover. The estimated ion energies are on the order of $80 \mathrm{eV}$, thereby producing films with a hydrogen content up to $40 \%$ (Dworschak 1990). This R.F. source allows lower ion energy densities than with the $2.45 \mathrm{GHz}$ source previously used by Dartois et al. (2005), giving a-C:H films with higher hydrogen content due to the decreased preferential sputtering of the $\mathrm{C}-\mathrm{H}$ bonds vs. the $\mathrm{C}-\mathrm{C}$ bonds. Lower pressures leading to higher ion energies also lead to sputtering of hydrogen. Samples are prepared at room temperature, although the substrate temperature has a strong influence on the film properties (Dworschak 1990).

To control the deposition rate for the films, we used the correlation between the optical depth of the $3.4 \mu \mathrm{m}$ band and the thickness of the films $(d)$ for films with $d>100 \mathrm{~nm}$, using their Fabry-Perot interference fringes that are due to internal reflections within the film itself. We extrapolated this correlation for films with $d<100 \mathrm{~nm}$ (see Appendix A for details). As a first estimate of the refraction index of these films in the optical, we used the relation in (Godard 2011) between the optical gap ( $\left.\mathrm{E}_{04}\right)$ and the refraction index measured for different $\mathrm{a}-\mathrm{C}: \mathrm{H}$ and $\mathrm{a}-\mathrm{C}$ (amorphous carbon) films (e.g., Dischler et al. 1983; Lazar 1998; Kassavetis et al. 2007). For our a-C:H materials, we derived the Tauc band gap $E_{\mathrm{g}}=2.5 \mathrm{eV}$, or $E_{04}=3.1 \mathrm{eV}$, and using the aforementioned relation, we estimate $n_{0} \sim 1.4-1.8$.

\subsection{Preparation of soot films}

Soot samples were produced at the Institut des Sciences Moléculaires d'Orsay (France) using the setup Nanograins (Pino et al. 2008; Carpentier et al. 2012). In the setup, whose scheme is shown in Fig. 2, a flat burner (McKenna) provides flames of premixed hydrocarbon and oxygen gas. Such a laminar flame is a one-dimensional chemical reactor offering a broad range of combustion conditions and sampling of byproducts. Here the pressure was maintained at 40 mbar by continuous pumping. The fuel used was ethylene $\left(\mathrm{C}_{2} \mathrm{H}_{4}\right)$ premixed with oxygen before flowing through the burner at a controlled flow rate

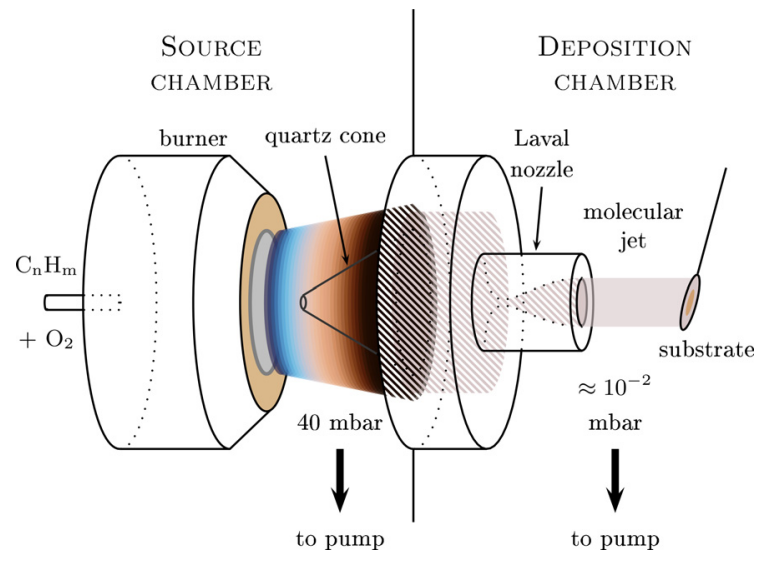

Fig. 2. Nanograins, an experimental setup for the preparation of soots. This scheme shows the burning region, the extraction cone in quartz, and the gas flow region shaped by a nozzle where the soot samples are collected by impaction on a window (Carpentier et al. 2012).

(4 l/min), and a $\mathrm{C} / \mathrm{O}$ ratio set to 1.05 . An $\mathrm{N}_{2}$ shield around the burning region was applied at a controlled flow rate of $31 / \mathrm{min}$. The byproducts were then extracted by a water-cooled quartz cone (hole diameter $=1 \mathrm{~mm}$ ) inserted into the flame at $30 \mathrm{~mm}$ from the burner. The burned gas and soot flow through a nozzle into a chamber at $5 \times 10^{-2}$ mbar. Soot particles were deposited on $\mathrm{MgF}_{2} / \mathrm{KBr} / \mathrm{NaCl}$ windows or $\mathrm{Au} / \mathrm{C} / \mathrm{Pt}$ membranes, inserted directly into the molecular flow. The thicknesses of the soot nanoparticle films varied in a range of a few tens of nm to $1 \mu \mathrm{m}$, depending both on the production rate and the exposure time, to optimize the spectral response in the different wavelength domains from the IR to the VUV.

\subsection{Instruments for UV and IR spectroscopy}

Ultraviolet measurements of our carbon analogs were performed with three instruments. At the IAS we use a UV-Vis spectrometer $(\lambda=210-710 \mathrm{~nm})$ with a sub-nm resolution. It consists of a deuterium lamp, whose light is conducted by optical fibers and illuminates the sample. The transmitted signal is then collected and carried by optical fibers toward a grating spectrometer (Maya PRO UV, Ocean Optics). For measurements in the vacuum ultraviolet, we use the APEX branch of the DISCO beamline at the SOLEIL synchrotron. The APEX chamber allows measurements at $\lambda=50-260 \mathrm{~nm}$ in windowless mode (Giuliani et al. 2009), with a beam size at the sample of $\leq 2 \mathrm{~mm}$. From now on, we interchange between the terms VUV and FUV to refer to this wavelength range. Finally, a spectrophotometer (Specord, Analytikjena) allowed measurements on $\mathrm{MgF}_{2}$ windows at $\lambda=190-1000 \mathrm{~nm}$.

Infrared measurements for the thicker $(d>300 \mathrm{~nm})$ films deposited on $\mathrm{MgF}_{2}$ windows were made using an evacuated FTIR spectrometer (Bruker Vertex 80V) equipped with a $\mathrm{KBr}$ beamsplitter and an MCT detector working in the 7000 to $400 \mathrm{~cm}^{-1}$ (2.5 to $15 \mu \mathrm{m})$ spectral range at $1 \mathrm{~cm}^{-1}$ resolution. To measure the infrared spectra of ultra-thin films $(d<100 \mathrm{~nm})$ we used the infrared microscope available at the SMIS beam line at the SOLEIL synchrotron. This microscope benefits from high synchrotron brilliance in the $2.5-100 \mu \mathrm{m}$ spectral range and a $10 \times 10 \mu \mathrm{m}$ spot size on the sample. 


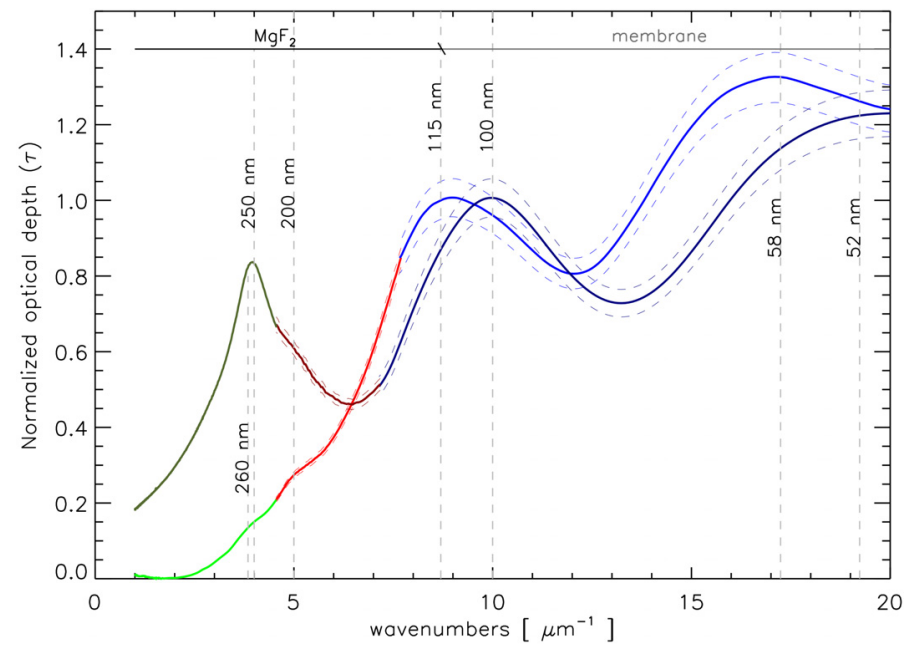

Fig. 3. VUV measurements of ultra-thin films: a-C:H and soot optical depth spectra normalized to $\tau\left(\sim 10 \mu \mathrm{m}^{-1}\right)$. a-C:H film, $-: \mathrm{MgF}_{2}$ (Specord), -: $\mathrm{MgF}_{2}$ (DISCO), -: TEM membrane (DISCO). Soot film, -: $\mathrm{MgF}_{2}$ (Specord), -: $\mathrm{MgF}_{2}$ (DISCO), -: TEM membrane (DISCO). Dashed lines show measurement dispersion.

\section{Measurements}

\subsection{UV spectra: a-C:H and soots}

Transmission spectra were obtained by correcting each spectral measurement by an electronic offset and then dividing the spectra of the desired carbon film deposited on the substrate $\left(\mathrm{MgF}_{2}\right.$ or a membrane) by the spectra of the substrate alone. We assumed that there are no optical effects due to the substrates, which we further discuss in Appendix B. The resulting normalized optical depth spectra for an a-C:H and a soot nanoparticle film are shown in Fig. 3.

The UV-VUV spectra reveal the electronic structure of the carbon analogs, arising from transitions between threefold ( $\mathrm{sp}^{2}$ bonding) and fourfold $\left(\mathrm{sp}^{3}\right)$ bonding coordination, and any mixture of these two bondings (sp bonding is not considered here as it is a minor component in our samples). The optical gap reaches from states in the valence $\sigma$ and $\pi$ band to states in the conduction $\sigma^{*}$ and $\pi^{*}$ bands. The $\pi-\pi^{*}$ bands due to $\mathrm{sp}^{2}$ sites typically occur from 200 to $500 \mathrm{~nm}\left(2-5 \mu \mathrm{m}^{-1}\right)$ and dominate the optical gap value. The $\sigma-\sigma^{*}$ transitions peak below $200 \mathrm{~nm}$ $\left(5-10 \mu \mathrm{m}^{-1}\right)$. Both of these bands produce an absorption from the UV to the visible and infrared domain. The optical gap in amorphous or disordered carbon can extend to the IR induced by defect states. It can take a value of $0 \mathrm{eV}$ for graphite, along a plane, and up to $5.5 \mathrm{eV}$ for diamond.

An a-C:H sample of thickness $\geq 30 \mathrm{~nm}$ becomes optically thick in the VUV. However, these films are optically thin in the UV-Vis range, which allows us to discern weaker absorption features, such as the absorption bands at $\sim 4-5 \mu \mathrm{m}^{-1}$. The reconstructed VUV spectra of an ultra-thin a-C:H film $(d \sim 20 \mathrm{~nm})$ and soot film $(d \sim 50 \mathrm{~nm})$ are shown in Figs. $4 \mathrm{a}$ and $\mathrm{b}$, respectively. Fitting parameters are given in Table 1.

For the a-C:H film, we identify four absorption features, fitted by Gaussian distributions. Two overlapping absorption peaks $(\mathrm{G} 1, \mathrm{G} 2)$ occur at $\sim 4$ and at $5 \mu \mathrm{m}^{-1}$. G1 is assigned to $\pi-\pi^{*}$ transitions due to locally aromatic carbon clusters (Robertson \& O'Reilly 1987). G2 is assigned to a superposition of carbon $\pi-\pi^{*}$ transitions and/or $n-\sigma^{*}$ transitions from oxygen contamination as discussed in Gadallah et al. (2011). The third absorption (G3) peak at $\sim 9 \mathrm{~m}^{-1}(115 \mathrm{~nm})$ in the far-UV

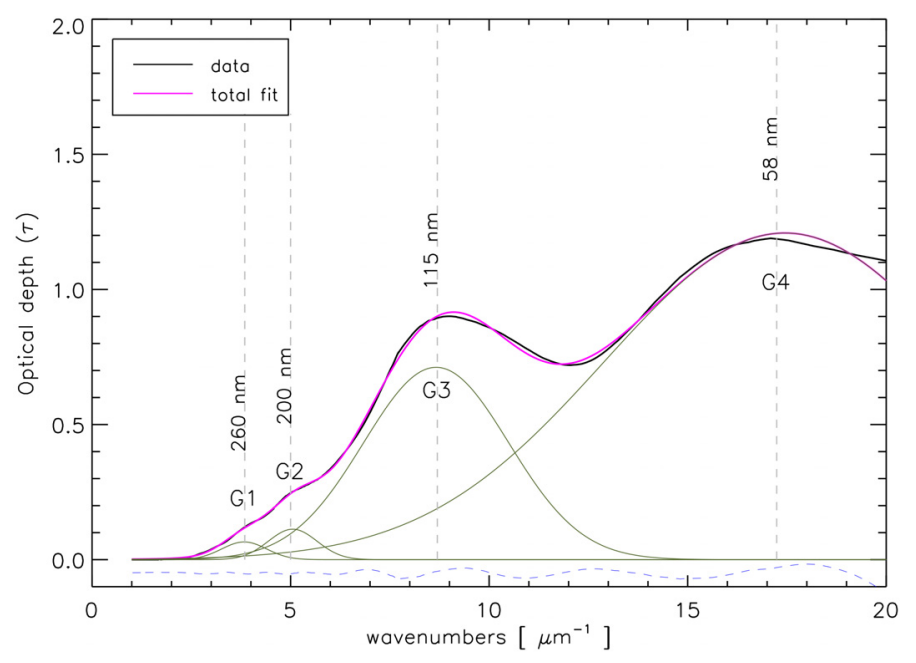

(a)

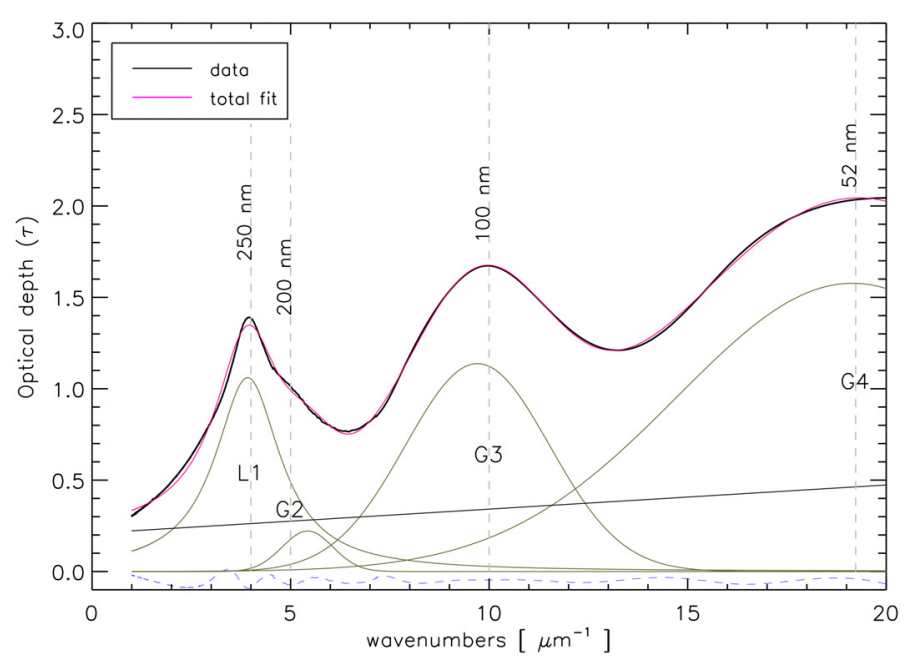

(b)

Fig. 4. VUV optical depth in the VUV of a) an a-C:H film and b) a soot nanoparticle film (described in the text). The deconvoluted spectral features are listed in Table 1.

Table 1. Best-fit parameters for VUV spectra.

\begin{tabular}{cccc}
\hline \hline Function & Parameter $\left[\mu \mathrm{m}^{-1}\right]$ & Soot & a-C:H \\
\hline \multirow{3}{*}{ Lorentzian (L1) } & area & 3.33 & \\
& center & 3.92 & \\
& width & 2.01 & \\
\hline \multirow{3}{*}{ Gaussian (G1) } & height & & 0.06 \\
& center & & 3.83 \\
& width & & 5.64 \\
\hline \multirow{3}{*}{ Gaussian (G2) } & height & 0.22 & 0.11 \\
& center & 5.42 & 5.05 \\
& width & 6.37 & 6.13 \\
\hline \multirow{3}{*}{ Gaussian (G3) } & height & 1.14 & 0.71 \\
& center & 9.71 & 8.67 \\
& width & 1.81 & 1.83 \\
\hline \multirow{3}{*}{ Gaussian (G4) } & height & 1.58 & 1.21 \\
& center & 19.2 & 17.4 \\
& width & 4.41 & 4.55 \\
\hline
\end{tabular}


is assigned to $\sigma-\sigma^{*}$ transitions. A fourth peak (G4) is found at $\sim 16 \mu \mathrm{m}^{-1}(58 \mathrm{~nm})$. A tentative identification of this fourth peak is given below.

The soot UV absorption spectrum was fitted by a Lorentzian, three Gaussians, and a linear function (slope $\sim 0.01$ in energy units). The first peak (L1) is well-adjusted by a Lorentzian function peaking at $3.92 \mu \mathrm{m}^{-1}$ and assigned to $\pi-\pi^{*}$ transitions. The relatively strong intensity of this feature points to a highly polyaromatic material, close in shape but not in position to the interstellar UV bump at $4.6 \mu \mathrm{m}^{-1}$. A weak peak (G2) at $5.4 \mu \mathrm{m}^{-1}$ is assigned to $\pi-\pi^{*}$ transitions from carbon and/or $n-\sigma^{*}$ transitions from oxygen contamination. A third broad peak (G3) occurs at $10 \mu \mathrm{m}^{-1}(100 \mathrm{~nm})$ and a fourth peak (G4) is present at about $19 \mu \mathrm{m}^{-1}(52 \mathrm{~nm})$. The linear function helps fit the soot opacity from $1 \mu \mathrm{m}^{-1}$ (while no linear function is required for the a-C:H fit).

The position of peaks in the soot film are found at higher energies than for the a-C:H, with $\Delta(\mathrm{L} 1-\mathrm{G} 1)=0.19 \mathrm{eV}, \Delta \mathrm{G} 2=$ $0.45 \mathrm{eV}, \Delta \mathrm{G} 3=1.62 \mathrm{eV}, \Delta \mathrm{G} 4=2.47 \mathrm{eV}$. Two $\pi$-electronic transitions are found between $200-260 \mathrm{~nm}$ in both materials. For the hydrogen-richer a-C:H, the lower $\mathrm{sp}^{2}$ carbon fraction decreases the $\pi$ density of states, which accounts for the weaker $\pi-\pi^{*}$ band strengths. By taking the optical depth ratio of the L1 to G3 feature in the soot spectra (Fig. 4a) and dividing it by the optical depth ratio of the G1 to G3 feature in the a-C:H spectra (Fig. 4b), we find that the $\pi-\pi^{*}$ resonance is $\sim 10$ times stronger in soot than in a-C:H.

Laboratory spectra of gas phase PAHs have shown their contribution to a FUV rise from a band with an extrapolated maximum at $\sim 17.7 \mathrm{eV}(70 \mathrm{~nm})$ (Joblin et al. 1992). Later on, Malloci et al. (2004) computed the absolute photo-absorption cross sections of 20 PAHs in the VUV. They found a good agreement both for the lower-lying bands of $\pi-\pi^{*}$ character and for the collective single broad absorption peak at $\sim 17-18 \mathrm{eV}$, composed of $\sigma-\sigma^{*}$, $\pi-\sigma^{*}, \sigma-\pi^{*}$ and Rydberg state transitions, with possible contributions from collective effects (e.g., plasmons). In graphite, in addition to the $\sigma$ and $\pi$ interband transitions, a band at $27 \mathrm{eV}$, measured in reflectance, was assigned to the collective excitations of the $\pi+\sigma$ plasmon (Djurišić \& Li 1999). In diamondlike carbon films (DLC), in addition to the $\sigma$ and $\pi$ interband transitions, a FUV contribution to absorption was detected using synchrotron ellipsometry, peaking at around $22 \mathrm{eV}(56 \mathrm{~nm}$, Franta et al. 2011, Fig. 5). The transitions in this range are attributed by the authors to valence $\sigma$ to extended states transitions $\sigma-\xi^{*}$. Figure 3 and Table 1 in Franta et al. (2011) depict the density of states for the corresponding band model. A marked double-peak structure was predicted by theoretical studies of the frequency-dependent dielectric permittivity in the optical to VUV domain of modeled carbon soot nanoparticles (Moulin et al. 2008; Langlet et al. 2009). The calculations predicted two peaks in the $\sim 20-30 \mathrm{eV}$ range. The double-peak structure was suggested to arise from the polyaromatic units with a size distribution ranging from a few angstroms up to a few $\mathrm{nm}$. In light of these findings, we tentatively assign the G4 band observed in both analogs to the features observed in this energy range in other carbonaceous materials. These transitions involve excitations from $\pi$ and $\sigma$ states toward extended states above the $\sigma^{*}$ band. The nature of these excited states is still unknown and work is in progress to explore it.

\subsection{Mid-infrared spectra: a-C:Hs and soots}

We measured the carbon analog thin films in the MIR with two objectives. The first is to correlate the integrated $3.4 \mu \mathrm{m}$ band to

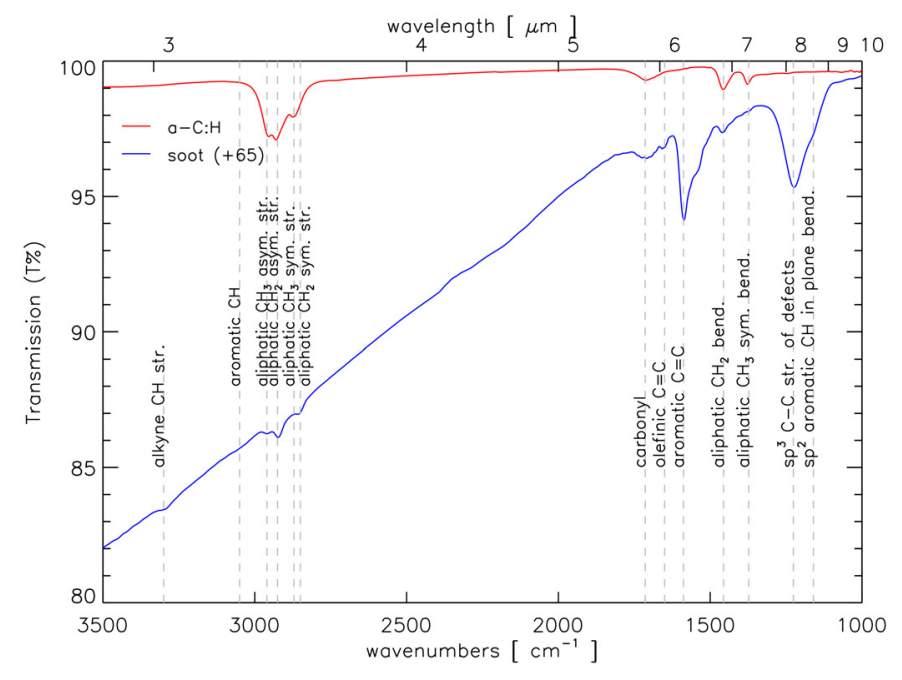

Fig. 5. Raw transmission spectra of an a-C:H film and a soot nanoparticle film (shifted by $+65 \%$ for comparison).

the thickness of an a-C:H film to normalize the corresponding laboratory UV spectra to interstellar extinction curves. The second objective is to assess the homogeneity of the films and compare spectra from the same spatial region where the UV spectra is measured. To this end, spectra with the SMIS FTIR microscope were taken every $0.2 \mathrm{~mm}$ across the ultra-thin a-C:H films deposited on $\mathrm{MgF}_{2}$ and on TEM grids to check the reproducibility of depositions.

Figure 5 shows the raw transmission mid-infrared spectra for an a-C:H and soot film. The continuum absorption is attributed to low-lying electronic transitions (Carpentier et al. 2012), while the superimposed bands are due to vibrational modes. The $\mathrm{CH}$ stretching bond region is seen at $2800-3100 \mathrm{~cm}^{-1}$. The bands between 1460 and $1380 \mathrm{~cm}^{-1}$ correspond to the aliphatic $\mathrm{CH}_{n}$ bending mode counterparts. The region between $1000-720 \mathrm{~cm}^{-1}$ corresponds to out-of-plane aromatic $\mathrm{CH}$ bending modes that are non-detectable due to the low amount of $\mathrm{sp}^{2} \mathrm{CH}$. Carbonyl is detected at $\sim 1718 \mathrm{~cm}^{-1}$, mainly due to oxygen contamination. A weak $\mathrm{OH}$ band is present at $\sim 3400-3500 \mathrm{~cm}^{-1}$.

The intensity of the $3.4 \mu \mathrm{m}$ band compared to the 6.2 and $8 \mu \mathrm{m}$ bands is much lower for soots than for the a-C:H, where aliphatic features dominate. For the a-C:H films we produce $\mathrm{H} / \mathrm{C} \sim 1$, while for soots, the $\mathrm{H} / \mathrm{C}$ ratio is lower $(\sim 0.01)$. The soot spectrum shows a $6.2 \mu \mathrm{m}$ band (at $1584 \mathrm{~cm}^{-1}$ ), due to the $\mathrm{C}=\mathrm{C} \mathrm{sp}{ }^{2}$ stretching modes. The $8.2 \mu \mathrm{m}$ band (at $\left.1225 \mathrm{~cm}^{-1}\right)$ is due to $\mathrm{C}-\mathrm{C}\left(\mathrm{sp}^{3}\right)$ stretching modes probably arising from defects, with a contribution at $1160 \mathrm{~cm}^{-1}$ due to $\mathrm{sp}^{2}$ aromatic $\mathrm{CH}$ bending modes. An alkyne $\mathrm{CH}$ stretching mode is present for soots at $3300 \mathrm{~cm}^{-1}$ and is non-detectable for the a-C:H. Band positions are coherent with previous laboratory soot infrared spectra (Pino et al. 2008; Carpentier et al. 2012).

Figure 6a shows the deconvolution of the vibrational modes of the $3.4 \mu \mathrm{m}$ band for a-C:H as in Dartois et al. (2004b). The $-\mathrm{CH}_{2}$ and $-\mathrm{CH}_{3}$ groups dominate the spectrum of the band at $3.4 \mu \mathrm{m}$, but the shoulder at $\sim 3050 \mathrm{~cm}^{-1}$ indicates the presence of olefinic and aromatic $\mathrm{C}-\mathrm{H}$ bonds.

The observed interstellar $3.4 \mu \mathrm{m}$ band in the diffuse medium and the measured IR spectra of the produced a-C:H films agree well (Dartois et al. 2005, 2007; Godard \& Dartois 2010). We used the oscillator strength of the observed modes to determine the aliphatic $\mathrm{CH}_{2} / \mathrm{CH}_{3}$ ratio of our a-C:H films 


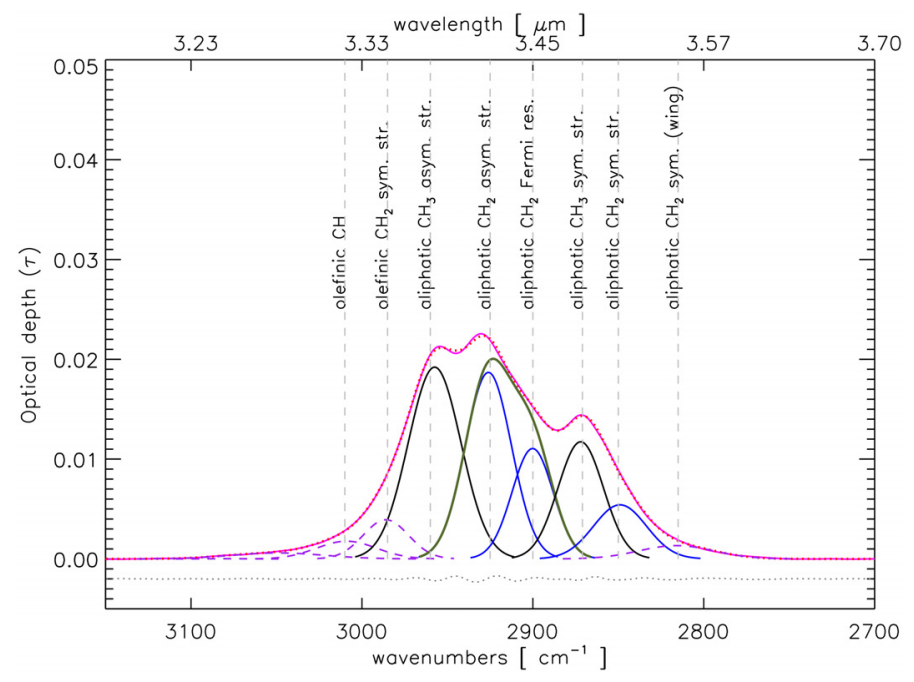

(a)

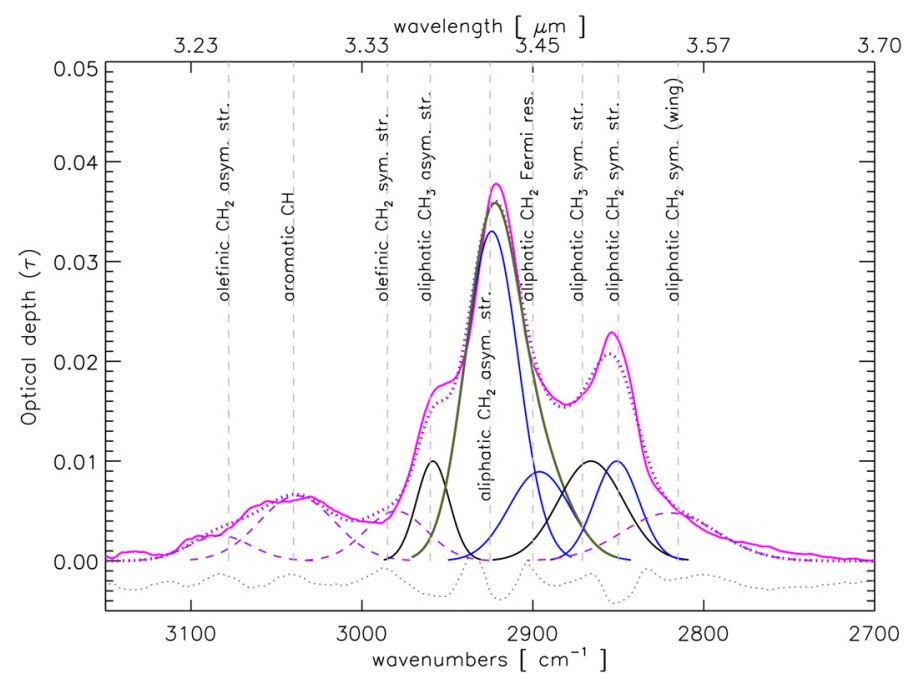

(b)

Fig. 6. Gaussian deconvolution of the $3.4 \mu \mathrm{m}$ band of an a) a-C:H film and b) soot film. The black lines correspond to the $-\mathrm{CH}_{3}$ groups, the blue lines to the $-\mathrm{CH}_{2}$ groups. The fit residual is in gray.

(Dartois et al. 2004a, 2007). The oscillator strength for the $\mathrm{CH}_{3}$ antisymmetric mode, at $3.38 \mu \mathrm{m}, \mathrm{A}\left(\mathrm{a}-\mathrm{CH}_{3}\right)=12.5 \times 10^{-18}$ $\mathrm{cm} /$ group, while for $\mathrm{CH}_{2}$ antisymmetric + Fermi resonance at $3.377 \mu \mathrm{m}, \mathrm{A}\left(\mathrm{a}-\mathrm{CH}_{2}\right)=8.4 \times 10^{-18} \mathrm{~cm} /$ group (Dartois et al. 2004a, 2007). The calculated $N\left(\mathrm{CH}_{2}\right) / N\left(\mathrm{CH}_{3}\right)$ ratio obtained from fitting the vibrational modes of the $3.4 \mu \mathrm{m}$ band is $2.4 \pm 0.3$, in agreement with observations toward Cyg OB2 No. 12, which samples only diffuse ISM dust (Pendleton \& Allamandola 2002), where a ratio of $2.5 \pm 0.4$ is found, and to observations toward the infrared galaxy IRAS $08572+3915$ (Dartois et al. 2007).

Figure $6 \mathrm{~b}$ shows the deconvolution of the vibrational modes of the $3.4 \mu \mathrm{m}$ band for the soot nanoparticle film. From this, the calculated aliphatic $N\left(\mathrm{CH}_{2}\right) / N\left(\mathrm{CH}_{3}\right)$ ratio $=7 \pm 3$. Using the oscillator strength for the aromatic $\mathrm{CH}$ vibrational mode at $3.289 \mu \mathrm{m}, A(\mathrm{CH})=1.9 \times 10^{-18} \mathrm{~cm} /$ group (Joblin et al. 1994), we find $N(\mathrm{CH})_{\text {arom }} / N\left(\mathrm{CH}_{2,3}\right)_{\text {aliph }}=1.1 \pm 0.3$, in agreement with Carpentier et al. (2012). Using the band strengths from methyl-substituted PAHs (Yang et al. 2013), we find $N(\mathrm{CH})_{\text {arom }} / N\left(\mathrm{CH}_{2,3}\right)_{\text {aliph }}=0.96 \pm 0.2$.

\section{VUV optical constants}

Optical constants were extracted from the transmission spectra of a-C:H films of varying thickness. For the soot film, a thickness $\sim 50 \mathrm{~nm}$ was estimated directly via AFM. From the transmission spectra, we obtained first-order values for the imaginary index of refraction, $k(\bar{v})$, according to the Beer-Lambert law,

$k \sim \frac{\tau(\bar{v})}{4 \pi \bar{v} d}$,

where $\tau$ is the optical depth, $v$ is in wave number units, and $d$ is the sample thickness. Before this, a baseline for the interference fringes due to multiple internal reflections was removed. By fitting the absorption in $k$ by a series of Gaussian profiles, $k$ was then extrapolated to $0 \mathrm{eV}$ and to higher energies, to apply the Kramers Kronig relations, closure relations linking the real and imaginary parts of the complex dielectric index of refraction (Bohren \& Huffman 1998). To derive the real part of the complex index of refraction from our absorption data, we used the subtractive Kramers-Kronig (SKK) method (Warren 1984). In this method, we fixed $n\left(v_{0}\right)$, known as the anchor point, a known value for the index of refraction at a reference frequency far from any strong absorption in the material considered, generally in the NIR-visible. SKK is the preferred method if $k$ is only known over a limited interval and the integrals are evaluated numerically. For the real index,

$n(v)=1+\frac{2}{\pi} P \int_{0}^{\infty} \frac{v^{\prime} k\left(v^{\prime}\right)}{v^{\prime 2}-v^{2}} \mathrm{~d} v^{\prime}$,

where $P$ denotes the Cauchy principal value of the integral. Inversely, for the imaginary index,

$k(v)=\frac{2 v}{\pi} P \int_{0}^{\infty} \frac{n\left(v^{\prime}\right)}{v^{2}-v^{\prime 2}} \mathrm{~d} v^{\prime}$.

For improved accuracy we used the formulation in Trotta \& Schmitt (1996), which reduces the error by using a finite wavelength integration range,

$n(v)=n\left(v_{0}\right)+\frac{2}{\pi} P \int_{v_{1}}^{v_{2}}\left[\frac{k\left(v^{\prime}\right) v^{\prime}-k(v) v}{v^{\prime 2}-v^{2}}-\frac{k\left(v^{\prime}\right) v^{\prime}-k\left(v_{0}\right) v_{0}}{v^{\prime 2}-v_{0}^{2}}\right] \mathrm{d} v^{\prime}$.

For a-C:H we took $n_{0}=1.5$ as an anchor point at $1 \mu \mathrm{m}$. For soot we took $n_{0}=1.7$ (Schnaiter et al. 1998). The Kramers-Kronig numerical integration calculates the desired $n$ value over the input energy range. Subsequently, the complex index of refraction values were used to numerically simulate a synthetic transmission value at each wavelength. For this, we used the model of Swanepoel (1983), who derived the expression for the transmission of a thin absorbing film on a transparent substrate $\left(\mathrm{MgF}_{2}\right.$ in our case). We constructed a real index template for a substrate made of $\mathrm{MgF}_{2}$ from $120-1000 \mathrm{~nm}$. For $\lambda=50-120 \mathrm{~nm}$ an inversion was made, keeping a constant $n$ value, but eventually, a more sophisticated scattering model should take into account the effects of a non-transparent substrate. The difference between the synthetic and measured transmission was calculated at each point until the difference between the observed and synthetic spectra was minimized, which occurred after a few iterations.

The optical constants derived for the laboratory a-C:H and soot films are shown in Figs. 7a and b. For a-C:H the systematic uncertainty is mostly due to the thickness estimated from the $n^{*}$ thickness vs. $3.4 \mu \mathrm{m}$ correlation (see Appendix A), where uncertainties in the thickness of the ultra-thin film go up to $\sim 50 \%$ 


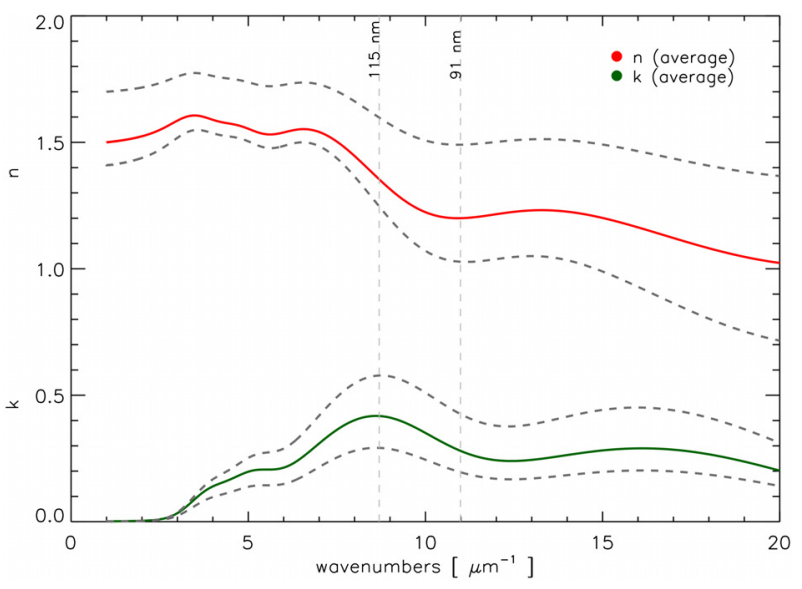

(a)

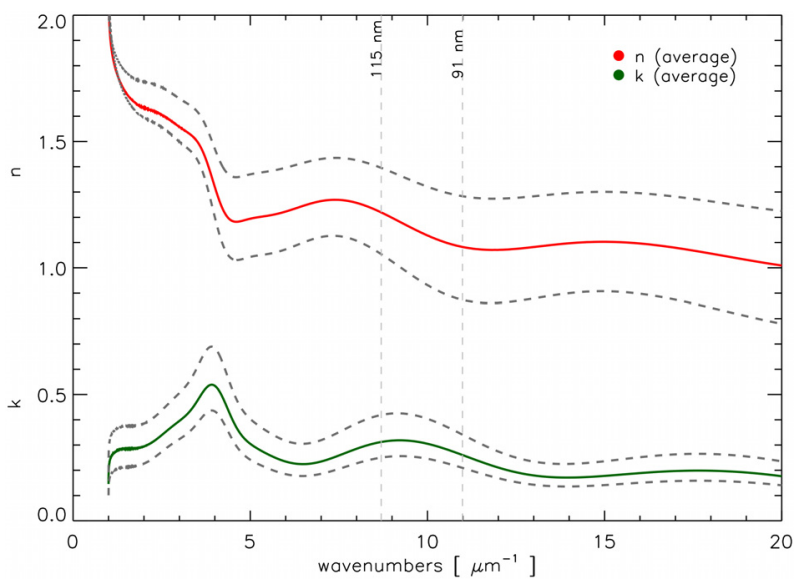

(b)

Fig. 7. Derived optical constants for a) an a-C:H film and b) a soot nanoparticle film, measured down to $\lambda=50 \mathrm{~nm}$. The dotted lines represent the compounded error bars dominated by uncertainty in the thickness and anchor points $\left(n_{0}\right)$.

for a-C:H. The uncertainty for the soot film thickness is as large as $\sim 25 \%$ (estimated from AFM measurements). The imaginary index of refraction reflects the major absorptive features seen in the laboratory spectra, that is, two FUV bumps for both materials at $\geq 10 \mu \mathrm{m}^{-1}$ and a Lorentzian peak at $\sim 4 \mu \mathrm{m}^{-1}$ for the soot. The uncertainty in the thickness measurement dominates the systematic uncertainty, demonstrating the experimental difficulty of measuring ultra-thin films in the VUV. As in Franta et al. (2011), who measured the optical properties of DLC films by ellipsometry in the $5-30 \mathrm{eV}$ range, the present study shows that direct optical measurements (e.g., in transmission) are necessary to remove ambiguities in calculating optical constants in the VUV, especially concerning the contribution of the band at $\sim 22 \mathrm{eV}$, which are expected by the models described in Sect. 3.1.

\section{Astrophysical implications}

Fitzpatrick \& Massa (2007) analyzed 328 Galactic interstellar extinction curves from the UV to the IR. Figure 8 shows two selected extinction curves (HD 21007 and ALS8107) which may represent a-C:H and soot components. The laboratory a-C:H spectrum is scaled to its laboratory $3.4 \mu \mathrm{m}$ optical depth and to the expected observational ratio of 250 toward the Galactic

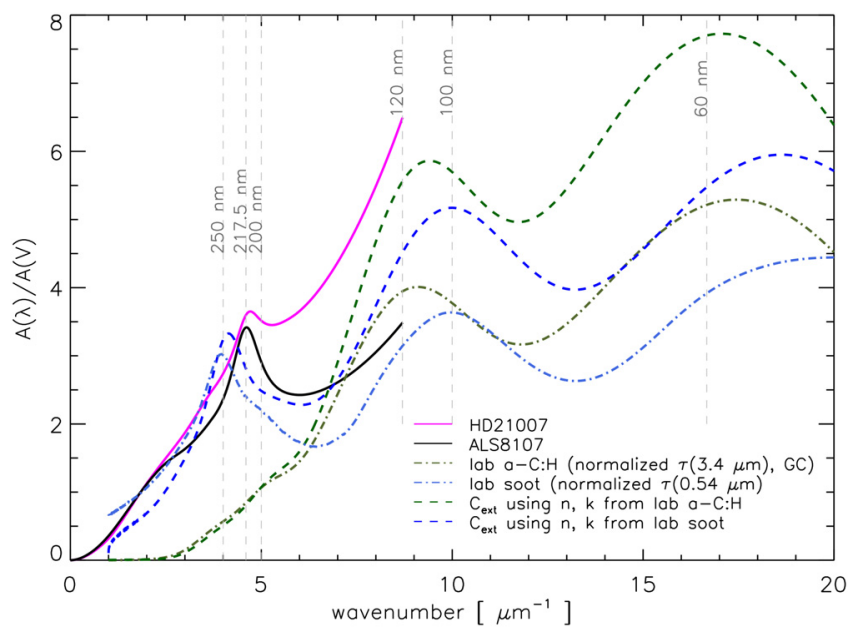

Fig. 8. Normalized extinction, $A(\lambda) / A(V)$, of representative lines of sight from Fitzpatrick \& Massa (2007; solid purple and black). Laboratory spectra for an a-C:H re-normalized to the optical depth of the observed $3.4 \mu \mathrm{m}$ band (dotted green) and renormalized laboratory soot spectra to its $V$-band optical depth (dotted blue). Calculated extinction curves from laboratory derived optical constants for an a-C:H (dashed green curve) and a soot (dashed blue).

center (Fig. 8). Observations of the optical depth of the $3.4 \mu \mathrm{m}$ band have been compared to the variations in visual extinction, $A_{V}$, toward Galactic sources and diffuse ISM lines of sight, and a correlation between these two has been found (Sandford et al. 1995; Godard 2011). A ratio of $A_{V} / \tau_{3.4} \sim 250$ has been estimated for lines of sight of the local ISM, while observations toward the Galactic center give a ratio of $A_{V} / \tau_{3.4} \sim 150$ (Sandford et al. 1995; Rawlings et al. 2003), although the difference is probably lower (Godard et al. 2011). The soot optical depth spectrum (Fig. 8) is scaled to its optical depth in the $V$-band $(551 \mathrm{~nm})$.

We used the laboratory optical constants obtained in Sect. 4 to calculate the extinction, $Q_{\text {ext }}$, that is due to a distribution of grain sizes, computed using the Mie formulae (Bohren \& Huffman 1998). Our primary objective is to illustrate the effect of the laboratory optical constants from this study on extinction curves. As a first attempt, we took into account a simple size distribution for Very Small Grains (VSGs) from Desert et al. (1990), that is,

$n(a) \propto a^{-p}$

where $p=2.6$, and the grain sizes varied between $a_{\min }=1$ to $a_{\max }=15 \mathrm{~nm}$. The total extinction is then calculated as

$C_{\text {ext }}\left[\mathrm{cm}^{-1}\right] \propto \int_{a_{\min }}^{a_{\max }} \pi a^{2} n(a) Q_{\mathrm{ext}} \mathrm{d} a$.

This analysis is limited to spherical, homogeneous, optically isotropic particles, and the resulting extinction is given by the green and blue dashed curves in Fig. 8. These optical constants should be implemented in more sophisticated extinction models. The calculated extinction shows that both materials present a steep FUV rise. However, the position of the FUV rise is primarily dictated by the position of the maximum of the $\sigma-\sigma^{*}$ band. For a-C:H band maximum positions occur at lower energies than for soots, which dominate the steep FUV rise. The UV peak of the calculated extinction curve calculated for laboratory soots is closer in position to the average galactic extinction UV bump at $4.6 \mu \mathrm{m}^{-1}$ (Fitzpatrick \& Massa 2007). 
Draine \& Lee (1984) suggested that crystalline graphite particles could be a carrier of the UV bump, but due to the difficulty of condensing graphite in space and the need for specific size distributions for spectral agreement (Czyzak et al. 1981), other carriers for this bump were sought. Mennella et al. (1996, 1999) and later Gadallah et al. (2011) showed that an increase of the $\mathrm{sp}^{2}$ carbon fraction as a result of UV irradiation of their carbon materials could give rise to the UV bump. Considering the mid-infrared and UV features, their samples are more comparable to our soot than to our a-C:H sample. Li et al. (2008) used buckyonions to reproduce observed interstellar extinction curves. Jäger et al. (1998) synthesized carbon analogs by pyrolizing cellulose materials and later by laser ablation of graphite (Jäger et al. 2008). However, their onion-like carbon nanoparticles did not show a strong UV bump. Polycyclic aromatic hydrocarbons (PAH) were proposed to simultaneously contribute to the $217.5 \mathrm{~nm}$ bump and the FUV rise (Malloci et al. 2004; Joblin et al. 2009). Steglich et al. (2010, 2013) suggested that PAHs with sizes around 50-60 carbon atoms per molecule could be responsible for the UV bump through a heterogenous distribution of sizes and not through single isolated species. CecchiPestellini et al. (2008) showed that these two spectral features and their variations can be accounted for by mixtures of PAHs in different ionization states. More recently, hydrogenated amorphous carbon grains, believed to be more easily formed in carbon star atmospheres, have been considered as potential carriers of the UV bump, particularly when irradiated by UV photons (Mennella et al. 1996, 1997; Gadallah et al. 2011).

Li \& Greenberg (1997) modeled interstellar extinction by using a three-component dust model: silicates, amorphous carbons, and PAHs. Recent extinction models including only two components (silicates and a-C/a-C:H; Jones 2012a,b) have found good agreement with previous work. More importantly, they account for the FUV rise and the UV bump only by means of the size-dependent properties of the carbonaceous component. The calculated extinction curves (using a a size distribution of carbon dust grains characterized by our optical constants) show that soot-like nanograins could be an important contributor to the UV bump, while a-C:Hs can contribute more significantly to the steep FUV rise. However, other dust components (like silicates) should be considered in a complete model of the observed extinction.

\section{Conclusions}

We have described the preparation of laboratory a-C:H and soot nanoparticle analogs to dust found in the ISM. We characterized thin films $(d<100 \mathrm{~nm})$ of these materials to measure their transmission spectra in the $\lambda=1 \mu \mathrm{m}$ to $50 \mathrm{~nm}$ range. These curves can be decomposed into absorption features involving transitions from states in the valence $\pi$ band to states in the conduction $\sigma$ band around 5 and $10 \mathrm{eV}$, the position and relative intensity varying with the $\mathrm{sp}^{2}$ content of the material. The description of the measurements required adding another band to explain the absorption, which peaked in the $16-19 \mathrm{eV}$ range. This band is predicted by calculations and is present at varying levels in other carbonaceous materials. It involves interband transitions to extended states.

By combining UV measurements to IR measurements, we scaled the UV contribution to the $3.4 \mu \mathrm{m}$ band to compare the observed UV extinction to our laboratory a-C:H measurements. We derived the optical constants for a laboratory a-C:H and soot from several sets of transmission measurements at different thicknesses. We used these optical constants to calculate extinction curves that show that a-C:Hs contribute to the steep FUV rise, while soots are suitable carriers of the UV bump. Astronomical spectra of dust shortward of the Lyman limit are currently lacking, mostly due to the absorption lines of hydrogen and helium, which have large FUV and extreme-UV (EUV) effective absorption cross sections (Hawkins \& Wright 1991). However, in dusty regions (where the opacity due to the gas component may be lower than the dust opacity), the laboratory FUV spectral features of carbon dust may be observed.

Acknowledgements. We thank Christophe Sandt (SOLEIL) for his help with the infrared microscope at the SMIS beam line. VUV measurements were made at the DISCO beam line of the SOLEIL synchrotron radiation facility (projects: 20141074 and 20130778). This work has been supported by the French program "Physique et Chimie du Milieu Interstellaire", the ANR COSMISME project (grant ANR-2010-BLAN-0502). L.G. acknowledges support from the CNES post-doctoral fellowship program.

\section{References}

Allamandola, L. J., Tielens, A. G. G. M., \& Barker, J. R. 1985, ApJ, 290, L25

Bohren, C. F., \& Huffman, D. R. 1998, Absorption and Scattering of Light by Small Particles, Wiley Science Series (Weinheim: Wiley-VCH)

Carpentier, Y., Féraud, G., Dartois, E., et al. 2012, A\&A, 548, A40

Cecchi-Pestellini, C., Malloci, G., Mulas, G., Joblin, C., \& Williams, D. A. 2008, A\&A, 486, L25

Colangeli, L., Bussoletti, E., Pestellini, C. C., et al. 1997, Adv. Space Res., 20, 1617

Colangeli, L., Mennella, V., Blanco, A., et al. 1993, ApJ, 418, 435

Czyzak, S. J., Hirth, J. P., \& Tabak, R. G. 1981, Vistas Astron., 25, 337

Dartois, E., Muñoz Caro, G. M., Deboffle, D., Montagnac, G., \& D’Hendecourt, L. 2005, A\&A, 432, 895

Dartois, E., Marco, O., Muñoz-Caro, G. M., et al. 2004a, A\&A 423, 549

Dartois, E., Muñoz Caro, G. M., Deboffle, D., \& d'Hendecourt, L. 2004b, A\&A, 423, L33

Dartois, E., Geballe, T. R., Pino, T., et al. 2007, A\&A, 463, 635

Desert, F.-X., Boulanger, F., \& Puget, J. L. 1990, A\&A, 237, 215

Dischler, B., Bubenzer, A., \& Koidl, P. 1983, Appl. Phys. Lett., 42, 636

Djurišić, A. B., \& Li, E. H. 1999, J. Appl. Phys., 85, 7404

Draine, B. T. 2003, ARA\&A, 41, 241

Draine, B. T., \& Lee, H. M. 1984, ApJ, 285, 89

Duley, W. W. 1994, ApJ, 430, L133

Duley, W. W., \& Seahra, S. 1998, ApJ, 507, 874

Dworschak, W. 1990, Thin Solid Films, 189, 257

Fink, J., Müller-Heinzerling, T., Pflüger, J., et al. 1984, Phys. Rev. B, 30, 4713

Fitzpatrick, E. L., \& Massa, D. 2005, AJ, 130, 1127

Fitzpatrick, E. L., \& Massa, D. 2007, ApJ, 663, 320

Franta, D., Nečas, D., Zajíčková, L., Buršíková, V., \& Cobet, C. 2011, Thin Solid Films, 519, 2694

Furton, D. G., Laiho, J. W., \& Witt, A. N. 1999, ApJ, 526, 752

Gadallah, K. A. K., Mutschke, H., \& Jäger, C. 2011, A\&A, 528, A56

Gail, H.-P., Zhukovska, S. V., Hoppe, P., \& Trieloff, M. 2009, ApJ, 698, 1136

Giuliani, A., Jamme, F., Rouam, V., et al. 2009, J. Synchrotron Rad., 16, 835

Godard, M. 2011, Ph.D. Thesis, Université Paris Sud - Paris XI

Godard, M., \& Dartois, E. 2010, A\&A, 519, A39

Godard, M., Féraud, G., Chabot, M., et al. 2011, A\&A, 529, A146

Goto, M., Kwok, S., Takami, H., et al. 2007, ApJ, 662, 389

Greenberg, J. M., \& Chlewicki, G. 1983, ApJ, 272, 563

Greenberg, J. M., \& Li, A. 1999, Adv. Space Res., 24, 497

Hawkins, I., \& Wright, E. L. 1991, in Extreme Ultraviolet Astronomy, eds. R. F. Malina, \& S. Bowyer, 333

Henning, T., Jäger, C., \& Mutschke, H. 2004, in Astrophysics of Dust, eds. A. N. Witt, G. C. Clayton, \& B. T. Draine, ASP Conf. Ser., 309, 603

Henning, T., \& Salama, F. 1998, Science, 282, 2204

Jäger, C., Mutschke, H., \& Henning, T. 1998, A\&A, 332, 291

Jäger, C., Mutschke, H., Henning, T., \& Huisken, F. 2008, ApJ, 689, 249

Jäger, C., Mutschke, H., Henning, T., \& Huisken, F. 2011, in EAS Pub. Ser. 46, eds. C. Joblin, \& A. G. G. M. Tielens, 293

Jenniskens, P. 1993, A\&A, 274, 653

Joblin, C., \& Tielens, A. G. G. M. 2011, PAHs and the Universe: A Symposium to Celebrate the 25th Anniversary of the PAH Hypothesis, EAS Pub. Ser., 46 Joblin, C., Leger, A., \& Martin, P. 1992, ApJ, 393, L79 
L. Gavilan et al.: VUV spectroscopy of carbon dust analogs: contribution to interstellar extinction

Joblin, C., D’Hendecourt, L., Leger, A., \& Defourneau, D. 1994, A\&A, 281, 923

Joblin, C., Berné, O., Simon, A., \& Mulas, G. 2009, in Cosmic Dust - Near and Far, eds. T. Henning, E. Grün, \& J. Steinacker, ASP Conf. Ser., 414, 383

Jones, A. P. 2012a, A\&A, 540, A1

Jones, A. P. 2012b, A\&A, 540, A2

Jones, A. P., Ysard, N., Koehler, M., et al. 2014, Faraday Discussions, 168, 313

Kassavetis, S., Patsalas, P., Logothetidis, S., Robertson, J., \& Kennou, S. 2007, Diamond and Related Materials, 16, 1813

Langlet, R., Vanacharla, M., Picaud, S., \& Devel, M. 2009, J. Quant. Spectr. Rad. Transf., 110, 1615

Lazar, G. 1998, APPA, 100, 67

Leger, A., \& Puget, J. L. 1984, A\&A, 137, L5

Li, A., \& Draine, B. T. 2012, ApJ, 760, L35

Li, A., \& Greenberg, J. M. 1997, A\&A, 323, 566

Li, A., Chen, J. H., Li, M. P., Shi, Q. J., \& Wang, Y. J. 2008, MNRAS, 390, L39

Malloci, G., Mulas, G., \& Joblin, C. 2004, A\&A, 426, 105

Mennella, V., Colangeli, L., Palumbo, P., et al. 1996, ApJ, 464, L191

Mennella, V., Baratta, G. A., Colangeli, L., et al. 1997, ApJ, 481, 545

Mennella, V., Brucato, J. R., Colangeli, L., \& Palumbo, P. 1999, ApJ, 524, L71

Molster, F. J., \& Waters, L. B. F. M. 2003, in Astromineralogy, ed. T. K. Henning (Berlin: Springer Verlag), Lect. Notes Phys., 609, 121

Moulin, F., Devel, M., \& Picaud, S. 2008, J. Quant. Spectr. Rad. Transf., 109, 1791

Papoular, R., Conard, J., Guillois, O., et al. 1996, A\&A, 315, 222

Peeters, E., Hony, S., Van Kerckhoven, C., et al. 2002, A\&A, 390, 1089
Pendleton, Y. J., \& Allamandola, L. J. 2002, ApJS, 138, 75 Pino, T., Dartois, E., Cao, A.-T., et al. 2008, A\&A, 490, 665

Rawlings, M. G., Adamson, A. J., \& Whittet, D. C. B. 2003, MNRAS, 341, 1121

Robertson, J., \& O’Reilly, E. P. 1987, Phys. Rev. B, 35, 2946

Sandford, S. A., Pendleton, Y. J., \& Allamandola, L. J. 1995, ApJ, 440, 697

Schnaiter, M., Mutschke, H., Henning, T., et al. 1996, ApJ, 464, L187

Schnaiter, M., Mutschke, H., Dorschner, J., Henning, T., \& Salama, F. 1998, ApJ, 498, 486

Scott, A. D., Duley, W. W., \& Jahani, H. R. 1997, ApJ, 490, L175

Sloan, G. C., Jura, M., Duley, W. W., et al. 2007, ApJ, 664, 1144

Smith, F. W. 1984, J. Appl. Phys., 55, 764

Stecher, T. P., \& Donn, B. 1965, ApJ, 142, 1681

Steglich, M., Jäger, C., Rouillé, G., et al. 2010, ApJ, 712, L16

Steglich, M., Jäger, C., Huisken, F., et al. 2013, ApJS, 208, 26

Swanepoel, R. 1983, J. Phys. E Sci. Instr., 16, 1214

Tielens, A. G. G. M. 2008, ARA\&A, 46, 289

Tosatti, E., \& Bassani, F. 1970, Nuovo Cimento B Serie, 65, 161

Trotta, F., \& Schmitt, B. 1996, in NATO Advanced Study Institute on the Cosmic Dust Connection, 3rd Course of the International School of Space Chemistry, 179

van Diedenhoven, B., Peeters, E., Van Kerckhoven, C., et al. 2004, ApJ, 611, 928

Warren, S. G. 1984, Appl. Opt., 23, 1206

Yang, X. J., Glaser, R., Li, A., \& Zhong, J. X. 2013, ApJ, 776, 110

Zubko, V. G., Mennella, V., Colangeli, L., \& Bussoletti, E. 1996, MNRAS, 282, 1321 


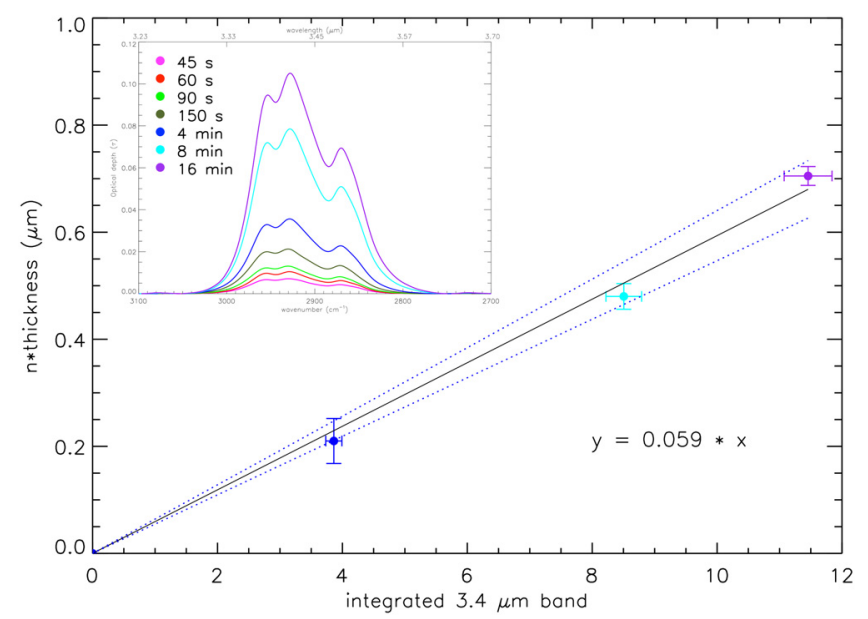

Fig. A.1. Correlation between $n *$ thickness of films $(d>300 \mathrm{~nm})$ vs. the integrated $3.4 \mu \mathrm{m}$ band area. This is used to estimate $n^{*}$ thickness for the ultra-thin films $(d<100 \mathrm{~nm})$ using their integrated $3.4 \mu \mathrm{m}$ values. The inset shows the $3.4 \mu \mathrm{m}$ band of a-C:H films produced with different deposition times.

\section{Appendix A: Thickness estimation of an a-C:H film}

The optical depth, $\tau$, obtained from transmission spectra, is equal to

$\tau=\alpha d$,

where $\alpha$ is the absorption coefficient, and $d$ is the film thickness. The absorption coefficient $\alpha$ is related to the imaginary part of the complex index of refraction, $k(v)$ by

$\alpha(v)=\frac{4 \pi \kappa(v)}{v}$,

which gives a first iteration of the $\kappa(v)$ index. From the Snell-Decartes law, we know that the optical path difference $\delta$ between a beam traversing the film and being reflected over each face of the film is

$\delta=2 n_{0} d \cos \left(\theta_{\mathrm{f}}\right)$.

The interference fringes of the film will have a maximum when

$\delta=m \lambda=\frac{m+1}{\Delta \sigma}$,

where $\Delta \sigma$ is the interfringe in wave numbers. Combining the previous two equations, we find that the thickness of the film is given by

$d=\frac{1}{2 n_{0} \Delta \sigma}$

where $n_{0}$ is assumed to be wavelength invariant, which is reasonable for visible and near-infrared wavelengths.

Measurements in the mid-infrared (from 400 to $8000 \mathrm{~cm}^{-1}$ ) give a limit for the detectable half interfringe of $\sim 200 \mathrm{~nm}$. This means that the infrared interfringe of ultra-thin films is not measurable. We used the correlation between the integrated $3.4 \mu \mathrm{m}$ band and $n *$ thickness (from the interfringe measurements of the thicker films, from Eq. (A.5)) to estimate the thickness for the ultra-thin films. Figure A.1 shows this correlation fitted by a linear function $y=m * x$, where $m=0.059 \pm 0.01$, along with $3 \sigma$ error bars.

A106, page 10 of 10

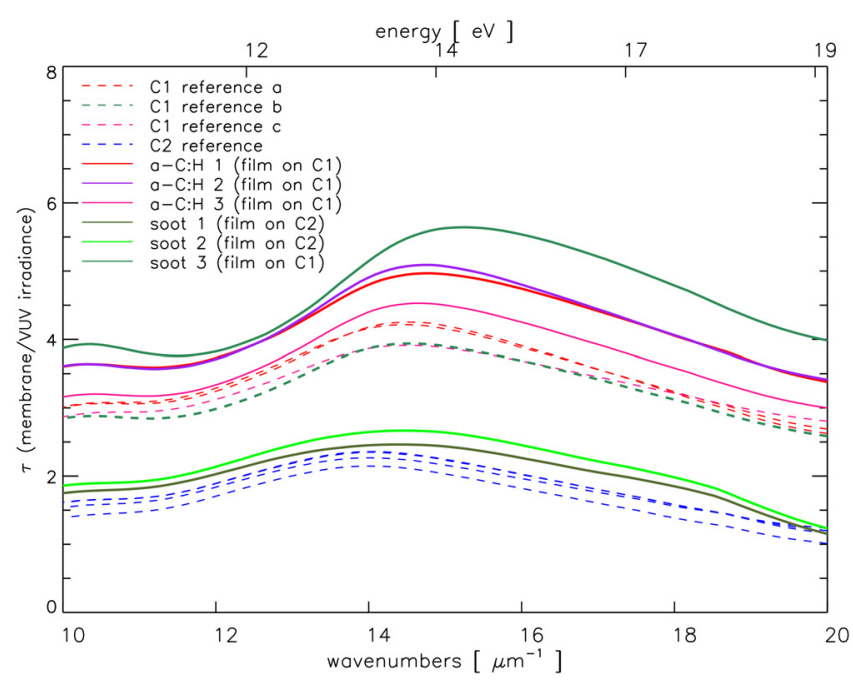

Fig. B.1. Transmission spectra of different carbon membranes (10-25 nm thick) used for VUV measurements (dashed lines), and carbon membranes $+\mathrm{a}-\mathrm{C}: \mathrm{H}$ or soot deposits (solid lines).

\section{Appendix B: Substrates in the VUV}

To study the influence of the substrate on the transmission spectra of a-C:H and soot films in the VUV, we studied the spectral dispersion of the substrates and the films deposited on such substrates, seen in Fig. B.1. These spectra are divided by the scaled spectral irradiance of the VUV beam. Spectra of substrate $\mathrm{C} 1$ (thickness $=15-25 \mathrm{~nm}$ ) show very low dispersion $(<0.01 \Delta \tau)$ for consecutive measurements $(\mathrm{C} 1$ a) and low dispersion $(<0.1 \Delta \tau)$ between two series of measurements. Spectra of reference $\mathrm{C} 2$ (thickness $\sim 10 \mathrm{~nm}$ ) show low dispersion $(<0.2 \Delta \tau)$ between consecutive measurements. The films (a-C:H or soots) deposited on $\mathrm{C} 1$ or $\mathrm{C} 2$ show the expected increased optical depth with characteristic peak positions different from the substrate. Division by the respective substrate gives us the spectra in transmission for such films, as shown in Figs. 3, 4. 